\title{
A GRAMMAR AND A PARSER FOR SPONTANEOUS SPEECH
}

\author{
Mikio Nakano, Akira Shimazu, and Kiyoshi Kogure \\ NTT Basic Research Laboratories \\ 3-1 Morinosato-Wakamiya, Atsugi-shi, Kanagawa, 243-01 Japan \\ \{nakano, shimazu, kogure\}Qatom.ntt.jp
}

\begin{abstract}
This paper classifies distinctive phenomena occurring in Japanese spontaneous speech, and proposes a grammar and processing techniques for handling them. Parsers using a grammar for written sentences cannot deal with spontaneous speech because in spontaneous spcech there are phenomena that do not occur in written sentences. A grammas: based on analysis of transcripts of dialogues was therefore devcloped. It has two distinctive features: it uses short units as input units instead of using sentences in grammars for written sentences, and it covers utterances including phrases peculiar to spontaneous speech. Sincc the grammar is an augmentation of a grammar for written sentences, it can also be used to analyze complex utterances. Incorporating the grammar into the distributed natural language processing model described elsewhere enables the handling of utterances includ ing variety of phenomena peculiar to spontaneous speech.
\end{abstract}

\section{INTRODUCTION}

Most dialogue understanding studies have focused on the mental states, plans, and intentions of the participants (Cohen et al., 1990). These studies have presumed that utterances can be analyzed syntactically and semantically and that the representation of the speech acts performed by those utterances can be obtained. Spontaneously spoken utterances differ considerably from written sentences, however, so it is not possible to analyze them syntactically and semantically when using a grammar for written sentences.

Spontaneous speech, a sequence of spontancously spoken utterances, can be distinguished from wellplanned utterances like radio nows and movie dialogues. Much effort has been put into incorporating grammatical information into speech understanding (e.g., Hayes et al. (1986), Young et al. (1989), Okada (1991)), but because this work has focused on wellplanned utterances, spontaneously spoken utterances have received little attention. This has partly been due to the lack of a grammar and processing technique that can be applicd to spontaneous speech. Consequently, to attain an understanding of dialogues it is necessary to develop a way to analyze spontaneous speech syntactically and semantically.

There are two approaches to developing this kind of analysis method: one is to develop a grammar and analysis method for spontaneous speech that do not depend on syntactic constraints as much as the conventional methods for written sentences do (Den, 1993), and the other is to augment the grammar used for written sentences and modify the conventional analysis method to deal with spontaneous speech The former method would fail, however, when new information is conveyed in the utterances; that is, when the semantic characteristics of the dialogue topic are not known to the hearer. In such cases, even in a dialogue, the syntactic constraints are used for understanding utterances. Because methods that disregard syntactic constraints would not work well in these kinds of cases, we took the latter approach.

We analyzed morc than a hundred dialogue transcripts and classified the distinctive phenomena in spontaneous Japanese speech. To handle those phenomena, we develop a computational model called linsemble Model (Shimazu et al., 1993b), in which syntactic, semantic, and pragmatic processing modules and modules that do combination of some or all of those processing analyze the input in parallel and independently. Even if some of the modules are unable to analyze the input, the other modules still output their results. This model can handle various kinds of irregular expressions, such as case particle omission, inversions, and fragmentary expressions.

We also developed Grass-I (Grammar for spontaneous speech in Japanese), which cnables the syntactic and semantic processing modules of the Finsemble Model to deal with some of the phenomena peculiar to spontaneous speech. Since Grass- $J$ is an augmentation of a grammar used to analyze written sentences (Grat-J, Grammar for texts in Japanese), Grass-J-based parsers can be used for syntactically complex utterances.

There are two distinctive features of Grass-J. One is that its focus is on the short units in spontaneous speech, called utterance units. An uttcrance unit instead of a sentence as in Grat-J is used as a grammatical category and is laken as the start symbol. A Grass-J-based parser takes an utterance unit as input and outputs the representation of the speech act (illocutionary act) performed by the unit. The other distinctive feature is a focus on expressions peculiar to spontancous speech, and here we explain how to augment Grat-J so that it can handle them. Previous studies of spontancous speech analysis have focused mainly on repairs and cllipses (Bear et al., 1992; Langer, 1990; Nakatani \& Hirschberg, 1993; Otsuka \& Okada, 1992), rather than expressions peculiar to spontancous speech.

This paper first describes Grat-J, and then classifies distinctive phenomena in Japanese spontancous speech. It then describes Grass- $J$ and presents several analysis examples. 
1. Subcategorization rule

Rule for NP (with particle) -VP constructions

$\mathrm{M} \rightarrow \mathrm{CH}$

$\langle\mathrm{M}$ head $\rangle=\langle\mathrm{H}$ head $\rangle$

$\langle\mathrm{H}$ subcat $\rangle=\langle\mathrm{M}$ subcat $\rangle \cup\langle\mathrm{C}\rangle$

$\langle\mathrm{M}$ adjacent $\rangle=\mathrm{nil}$

$\langle\mathrm{H}$ adjacent $\rangle=$ nil

$\langle\mathrm{M}$ adjunct $\rangle=\langle\mathrm{H}$ adjunct $\rangle$

$\langle\mathrm{M}$ |exical $\rangle=$

$\langle M$ sem index $\rangle=\langle H$ sem index $\rangle$

〈M sem restric)

$=\langle C$ sem restric $\rangle \cup\langle\mathrm{H}$ sem restric $\rangle$

Symbols $\mathrm{M}, \mathrm{C}$, and $\mathrm{H}$ are not names of categories but variables, or identifiers of root nodes in the graphs representing feature structures. $M_{1} C_{1}$ and $H$ correspond to mother, complement daughter, and head daughter The head daughter's subcat feature value is a set of feature structures.

2. Adjacency rule

Rule for VP-AUXV constructions, NP-particle constructions, etc.

$\mathrm{M} \rightarrow \mathrm{A} \mathrm{H}$

$\langle\mathrm{M}$ head $\rangle=\langle\mathrm{H}$ head $\rangle$

$\langle M$ subcat $\rangle=\langle$ H subcat $\rangle$

$\langle H$ adjacent $\rangle=\langle A\rangle$

$\langle M$ adjacent $\rangle=-$ nil

$\langle\mathrm{M}$ adjunct $\rangle:=\langle\mathrm{H}$ adjunct $\rangle$

$\langle M$ lexical $\rangle=-$

$\langle M$ sem index $\rangle=\langle H$ sem index $\rangle$

$\langle\mathrm{M}$ sem restric $\rangle$

$=\langle$ sem restric $\rangle \cup\langle H$ sem restric $\rangle$

$M, \Lambda$, and $H$ correspond to mother, adjacent daughter and head daughter. The head daughter's adjacent feature value is unified with the adjacent daughter's feature structure.

3. Adjunction rule

Rule for modifier-modifiee constructions.

$\mathrm{M} \rightarrow \mathrm{AH}$

$\langle M$ head $\rangle=\langle\langle(H$ head $\rangle$

$\langle M$ subcat $\rangle=\langle$ H subcat $\rangle$

$\langle\mathrm{H}$ adjacent $\rangle=$ nil

$\langle$ A adjunct $\rangle=\langle H\rangle$

$\langle M$ lexical $\rangle=-\cdots$

$\langle M$ sem index $\rangle=\langle H$ sem index $\rangle$

(M sem restric)

$=\langle A$ sem restric $\rangle \cup\langle H$ sem restric $\rangle$

$M, A$, and $H$ correspond to mother, adjunct daughter (modifier), and head daughter (modifiee). The adjunct daughter's adjunct feature value is the feature structure for the head daughter.

Fig. 1: Phrase struchure rules in Grat-J

\section{A GRAMMAR FOR WRITTEN SENTENCES}

Grat-J, a grammar for written sentences, is a nuification granmar loosely based on Japanese phraso structure grammar (JPSC) (Gunji, 1986). Of the six phrase structure rules used in Grat-J, the three related to the discussion in the following sections are shown in I'ig. 1 in a I'A'I'R-II like notation (Shieber, 1986). I exical items are represented by foaturo structures and example of which is shown in lig. 2 .

Grat-J-based parsers generate semantic representa-

\footnotetext{
${ }^{1}$ Rales for relative clauses and for verb-phrase coordinations are not shown here.
}

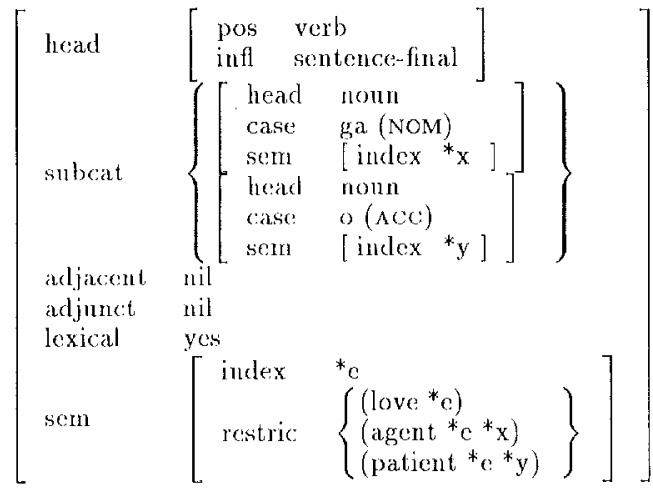

Fig. 2: Feature struclure for the word 'aisuru' (love).

tions in logical form in Davidsonian style. The semantic representation in each lexical item consists of a variable called an index (feature (sem index)) and restrictions placed on it (feature (sem restric)). Wvery time a plurase structure rule is applied, these restrictions are aggregated and a logical form is synthesized.

For example, let us again consider 'aisuru' (love). If, in the feature structure for the phrase 'Taro ga' (Taro-Nom), the (sem index) value is * ${ }^{*}$, and the (sem restric) value is $\left\{\left(\right.\right.$ taro $\left.\left.^{*} p\right)\right\}$, after the subcat cgorization rule is applied the (sem restric) value in the resulting foature structure for the phrase "laro git aisuru' ('laro loves) is $\left\{\left(\right.\right.$ taro $^{*} \mathrm{x}$ ) (love ${ }^{*}$ ) (agent, ${ }^{*}$ $\left.*_{x}\right)\left(\right.$ patient $\left.\left.*_{e}^{*} y\right)\right\}$.

Grat-J covers such fundamental Japanese phenomena as subcategorization, passivization, interrogalion, coordination, and negation, and also covers copulats, relative clauses, and conjunctions. We developed a parser based on (irat-J by using bottom-up chart. parsing (Kay, 1980). Unification operations are performed by using constraint projection, an efficient. method for unifying disjunctive feature descriptions (Nakano, 1991). The parser is implemented in Jancid Common Lisp ver. 4.0.

\section{DISTINCTIVE PHENOMENA IN JAPANESE SPONTANEOUS SI'EECH}

\section{3. $\mathrm{L}$ Classification of Phenomena}

Wo analyzed 97 telephone dialogues (about 300,000 bytes) about using $\left[A^{\prime} I_{S} X\right.$ to prepare documents and 26 dialogues (about 160,000 bytes) obtained from threc radio listener call-in programs (Shimazn et al., 1993a). We found that angnenting the grammars and analysis methods requires taking into account at least the following six phonomena in Japanese spontaneous specch.

(p1) expressions peculiar to Japanese spontaneous speech, including fillers (or hesitations).

(cx.) 'etto aru nclesukedomo ...' 'kono fairu tte ..' (well, we have them... this file is...)

(p2) particle (case particle) omission

(ex.) 'sore watashi yarimasu' (I will do it.)

(p3) main verb ellipsis, or fragmentary utterances 
(ex.) 'aa, shinkansen de Kyoto kara.' (uh, from Kyoto by Shinkansen line.)

(p4) repairing phrases

(ex.) 'ano chosya be, chosya no arufabetto jun ni naran da, indekkusu naai?' (well, are there, aren't there indices ordered alphabetically by authors' names?')

(p5) inversion

(ex.) 'kopii shite kudasai, sono ronbun.' (That paper, please copy.)

(p6) semantic mismatch of the theme/subject and the main verb

(ex.) 'rikuesuto no uketsuke jikan wa, 24-jikan jouji uketsuke teori masu.' ('The hours we receive your requests, they arc received 24 hours a day.)

\subsection{Treatment of the Phenomena by the Ensemble Model}

These kinds of phenomena can be handled by the Ensemble Model. As described in Section 1, the Ensemble Model has syntactic, semantic, and pragmatic processing modules and modules that do combination of some or all of those processings to analyze the in put in parallel and independently. Their output is unified, and even if some of the modules are unable to analyze the input, the other modules output their own results. This makes the Ensemble Model robust. Morcover, even if some of the modules are unable to analyze the input in real-time, the others output their: results in real-time.

The Ensemble Model has been partially implemented, and Ensemble/Trio-I consists of syntactic, semantic, and syntactic-semantic modules. It can handle (p2) above as described in detail elsewhere (Shimazu et al., 1993b). Phenomena (p3) through (p6) can be partly handled by another implementation of the Ensemble Model: Ensemble/QuartetI, which has pragmatic processing module as well as the three modules of Ensemble/'Trio-I. The pragmatic processing module uses plan and domain knowledge to handle not only well-structured sentences but, also ill-structured sentences, such as those including inversion and omission (Kogure et al., 1994).

To make the system more robust by enabling the syntactic and semantic processing modules to handle phenomena (p1) and (p3) through (p6), we incorporated Grass-J into those modules. Grass-J differs from Grat-J in two ways: Grass- $J$ has lexical entries for expressions peculiar to spontaneous speech, so that it can handle (p1). And because sentence boundaries are not clear in spontaneous speech, it uses the concept of utterance unit (Shimazu et al., 1993a) instead of sentence. This allows it to handle phenomena (p3) through (p6). For example, an inverted sentence can be handled by decomposing it, at the point where the inversion occurs, into two utterance units.

Fig. 3 shows the architecture of Lnsemble / Quartet I. Fach processing module is based on the bottomup chart analysis method (Kay, 1980) and a disjunctive feature description unification method called constraint projection (Nakano, 1991). The syntactic. semantic processing module uses Grass- $J$, the syntactic processing module uses Crass- $J$ without semantic constraints such as sortal restriction, the seman-
A: 1 anoo kisoken

well the Basic Research Labs.

cno ikikata o desune

to how to go ACC

'well, how to go to the Basic Research T,abs.'

13: 2 hai

uh-huh

'uh-hull'

A: 3 chotto shira nai nde

well know No' because

'because I don't know well'

4 oshie teitadaki taindesukedo

tell IIAVE-A-FAVOR want

'I'd like you tell me it'

F'ig. 4: Dialogue 1.

tic processing module uses Grass- / without syntactic constraints such as case information, and the pragmatic processing modulo uses a plan-based grammar.

\section{A GRAMMAR FOR SPONTANEOUS SPEECH}

This section describes Grass- $J$.

\subsection{Processing Units}

'Sentence' is used as the start symbol in grammars for written languages but sentenco boundaries are not. clear in spontaneous speech. 'Sentence' therefore cannot be used as the start symbol in grammars for spontancous specch. Many studies, though, have shown that utterances are composed of short units (Ievelt, 1989: pp. 23-24), that need not be sentences in written language. Grass-J uses such unit,s instead of sentences.

Consider, for example, ])ialogue 1 in Fig. 4. Ut. terances 1 and 3 cannot be regarded as sentences in written language. Let us, however, consider 'hai' in Utterance 2. It expresses participant B's confirmation of the contents of Utterance $1 .{ }^{2}$ Wach utterance in Dialogue 1 can thus be considered to be a speech act (Shimayu et al., 1993a). These utterances are processing units we call utterance units. 'They are used in Grass- $J$ instead of the sentences used in Grat-J. Ono feature of these units is that 'hai' can be interjected by the hearer at the end of the unit.

The boundaries for these units can be determined by using pauses, linguistic clues clescribed in the next section, syntactic form, and so on. In using syntactic form to determine utterance unit boundaries, Grass$J$ first stipulates what an utterance unit actually is. This stipulation is based on an investigation of dialogue transcripts, and in the current version of Grass. $J$, the following syntactic constituents are recognized as utterance units.

- verb phrases (including auxiliary verb phrases and adjective phrases) that may be followed by

\footnotetext{
"The roles of 'hai', an interjectory response corresponding to a back-channel utterance such as uh-huh in Finglish but which occurs more frequently in Japanese dialogue, are discussed in Shimazu et al. (1993a) and Katagiri (1993)
} 


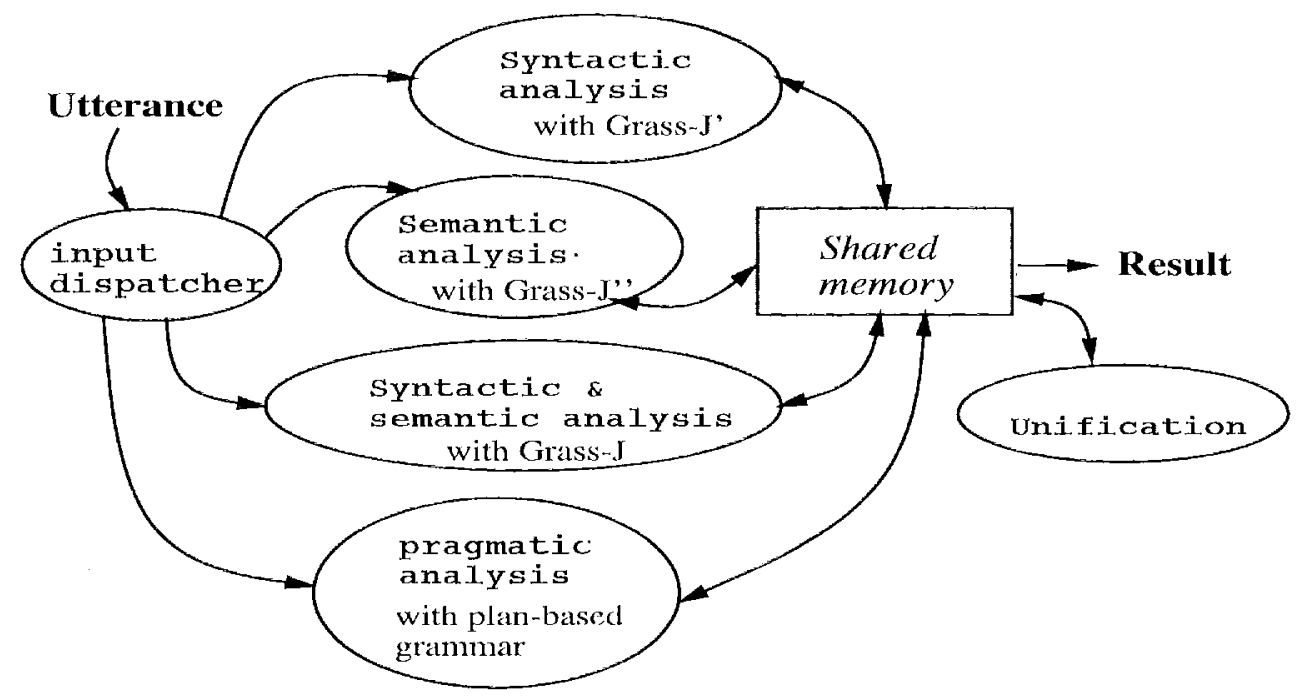

Fig. 3: Architecture of Ensernble/Quartet-I.

conjunctive particles and sentence-final particles - nomn phrases, which may be followed by particles

- interjections

- conjunctions

Grass-J includes a bundle of phrase structure rules used to derive speech act representation from the logical form of these constituents. A Grass-J-based parser inputs an utterance unit and outputs the representation of the speech act performed by the unit, which is then input to the discourse processing system.

Consider the following simple dialogue.
A: 1 genkou o
manuscript $A C C$
B: 2 hai
"Ihe manuscript"
uh-hull
"uh-huh"
A: 3 okut lekudasai send please 'please send me'

The logical form for Utterance 1 is ((manuscript $\left.{ }^{*} \mathrm{x}\right)$ ) so that its resulting speech act representation is

(1) $\left(\left(\operatorname{refer}_{\mathrm{e}}\right)\right.$ (agent $\left.*_{\mathrm{e}} *_{\mathrm{s}}\right)$ (speaker $\left.*_{\mathrm{s}}\right)$ (object $*_{0}$ $\left.*_{x}\right)\left(\right.$ manuscript $\left.\left.*_{x}\right)\right)^{3}$

or, as written in usual notation,

(2) Refer(speaker, ?x:manuscript(?x))

In the same way, the speech act representation for Utterance 3 is

(3) Request(speaker, hearer, send(hearer, speaker, ?y)).

The discourse processor would find that ? $\mathrm{x}$ in (2) is the same as ?y in (3). A detailed explanation of thi discourse processing is beyond the scope of this paper.

\footnotetext{
'Refer' stands for the surface referring in Allen and Perrault (1980)
}

\subsection{Treatment of Expressions Peculiar to Spontaneous Speech}

\section{Classification}

'I'he underlined words in Dialogue 1 in lig. 4 do not normally appear in written sentences. We analyzed the dialogue transcripts to identify expressions that, frequently appear in spoken sentences which includes spontaneous speech but that do not appear in written sentences, and we classified them as follows.

1. words phonologically different from those in written sentences (words in parenthesis are corresponding written-sentence words)

(ex.) 'shinakya' ('shinakercba', if someone does not do), 'shichau' ('shiteshimau', have done)

2. fillers (or hesitations such as well in linglish) (cx.) 'ctto', 'anoo'

3. particles peculiar to spoken language (ex.) 'tite', 'nante', 'toka'

4. interjectory particles (words inserted interjectorily after noun phrases and adverbial/adnominalform verb phrases) (ex.) 'ne', 'desune', 'sa'

5. expressions introducing topies (ex.) '(na)ndesukedo'; '(na)ndesuledomo', '(na)ndesuga'

6. words appearing after main verb phrases (these words take the sentence-final form of verbs/auxiliary verbs/adjectives)

(ex.) 'yo', 'ne', 'yone', 'keredo', 'kedo', 'keredomo', 'ga', 'kedomo', 'kara'

Nagata and Kogure (1990) addressed Japanese sentence-final expressions peculiar to spoken Japanese sentences but did not deal with all the spontaneous specch expressions listed above. 'These expressions may be analyzed morphologically (Takeshita \& Fukınaga, 1991). Because some expressions peculiar to spontancous speech do not affect the propositional 
content of the sentences, disregarding those expressions might be a way to process spontaneous speech. Such cascaded processing of morphological analysis and syntactic and semantic analysis disables the incremental processing required for real-time dialoguc understanding. Another approach is to treat these kinds of expressions as cxtra, 'noisy' words. Although this can be done by using a robust parsing technique, such as the one developed by Mellish (1989), it requires the sentcnce to be processed more than two times, and is therefore not suitable for real-time dialogue understanding. In Grass- $J$ these expressions are handled in the same way as expressions appearing in written language, so no special techniques are needed.

Words phonologically different from corresponding words in written-language

'The words 'teru' and 'ndesu' in 'shit teru ndesu ka' (do you know that?) correspond semantically to 'teiru' and 'nodesu' in written sentences. We investigated such words in the dialogue data (Fig. 5). One way to handle these words is to translate them into their corresponding written-language words, but because this requires several steps it is not suitable for incremental dialogue processing. We therefore regard these words as independent of their corresponding words in written-language, even though their lexical cntries have the same content.

\section{Fillers}

Fillers such as 'anoo' and 'etto', which roughly correspond to well in English, appear frequently in spontancous speech (Arita et al., 1993) and do not affect the propositional content of sentences in which they appear ${ }^{4}$. One way to handle them is to disregard them after morphological analysis is completed. As noted above, however, such an approach is not suitable for dialogue processing. We therefore treat them directly in parsing.

In Grass- $J$, fillers modify the following words, whatcver their grammatical categories are. 'I'he feature structure for fillers is as follows.

$\left[\begin{array}{ll}\text { head } & {[\text { pos interjection }]} \\ \text { subcat } & \{\} \\ \text { adjunct } & {[\text { lexical }+]} \\ \text { adjacent } & \text { nil } \\ \text { lexical } & + \\ \text { sem } & {[\text { restric }\{\}]}\end{array}\right]$

The value of the feature lexical is either + or - : it is + in lexical iterns and - in feature structures for phrases composed, by phrase structure rules, of subphrases. Because these words do not affect propositional contents, the value of the feature (sem restric) is compty.

For example, let us look at the parse tree for 'etto 400-yen desu' (well, it's 400 yen). Symbols I (Interjection), NP, and VP are abbreviations for the complex feature structures.

\footnotetext{
${ }^{4}$ Although Sadanobu and Takubo (1993) investigated the discourse management function of fillers, we do not discuss it here.
}

1. expressions related to aspects teku (teiku in written-language), teru (teiru), chau (tesiman), etc.

2. expressions related to topic marker 'wa' cha (tewa), chaa (tewa), ccha (tewa), ja (dewa), etc.

3. expressions related to conjunctive particle 'ba' nakerya (nakereba), nakya (nakereba), etc.

4. expressions related to formal nouns n (no), mon (mono), toko (tokoro), ete.

5. demonstratives kocchi (kochira), korya (korewa), so (sou), soshitara (soushitara), sokka (souka), socchi (sochira), son (sono), soreja (sorcdewa), sorejaa (soredewa), etc:

6. expressions related to interrogative pronoun nani nanka (nanika), nante (nanito), etc.

7. other mokkai (mouikkai), etc.

Fig. 5: Words that in spoken language differ from corresponding words in written language.

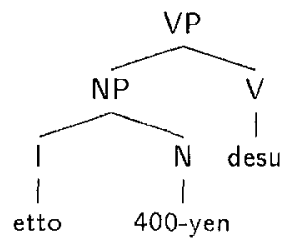

The filler 'etto' modifies the following word ' 400 yen' and the logical form of the sentence is the same as that of ' 100 -yen desu'.

\section{Particles peculiar to spoken language}

Words such as 'tte' in 'Kyoto tte Osaka no tsugi no eki desu yone' (Kyoto is the station next to Osaka, isn't it?) work in the same way as casc-marking/topicmarking particles. Because they have no corresponding words in written language, lexical entries for them are required. 'I'hese words do not correspond to any specific surface case, such as 'ga' and ' $O$ '. Jike the topic marker 'wa', the semant ic relationships they express depend on the moaning of the phrases they connect.

\section{Interjoctory particles}

Interjectory particles, such as 'ne' and 'desune', follow noun phrases and adverbial/adnominal-form verb phrases, and they do not affect the meaning of the utterances. 'The interjectory particle 'ne' differs from the sentence-final particle 'ne' in the sense that the latter follows sentence-final form verb phrases. These kinds of words can be treated by regarding them as particles following noun phrases and verbs phrases. The following is the feature structure for these words.

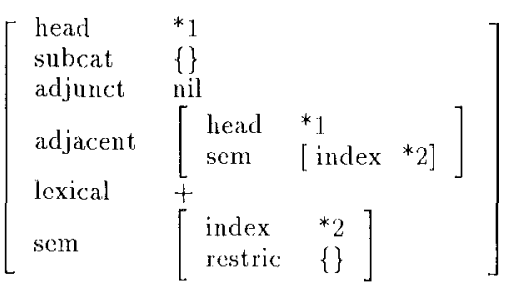


'I'he interjectory particles indicate the end of utterance units; they do not appear in the middle of utterance units. 'They function as, so to speak, utteranceunit-final particles. 'Therefore, a nom phrase followed by an interjectory particle forms a (surface) referring speoch act in the same way as noun phrase utterunces. Interjectory particles add nothing to logical forms. For example, the speech act representation of 'genkou o desune' is the same as (2) in Section 4.1.

\section{Expressions introducing topics}

As in Utterance 4 of Dialogue 1, an expression such as (na)ndesukedo(mo) frequently appears in dialogues, especially in the beginning. This expression introduces a now topic. One way to handle an expression such as this is to break it down into $n a+n d e s u+k e d o+$ mo. 'T'his process, however, prevents the system from detecting its role in topic introduction. We therefore consider each of these expressions to be one word. The reason these expression are used is to make a topic explicit, by introducing a discourse referent, (Thomason, 1990). Consequently, all 'introduce-topic' speech act is formed. These exmressions indicate the end of an utterance unit as an interjectory particle.

Words appearing after main verb phrase

It has already been pointed out that sentence-final particles, such as 'yo' and 'nc', frequently appear in spoken Japanose sentencos (Kawamori, 1991). ("onjunctive particles, such as 'kedo' and 'kara', are also used as sentence-final particles (Hosaka et al., 1991) and they are treated as such in Grass-J. They perform the function of anticipating the hearer's reaction, as a trial expression docs (Clark \& Wilkes-Gibbs, 1990). They also indicate the end of utterance units.

\section{ANALYSIS EXAMPLES}

Below we show results obtained by using a Crass$J$-based parser to analyze some of the utterances in Dialogue 1. UU means the utterance unit category.

- Utterance 1: 'anoo kisoken eno ikikata o desune' (well, how to go to the Basic Rescarch I aalss.) parse tree:

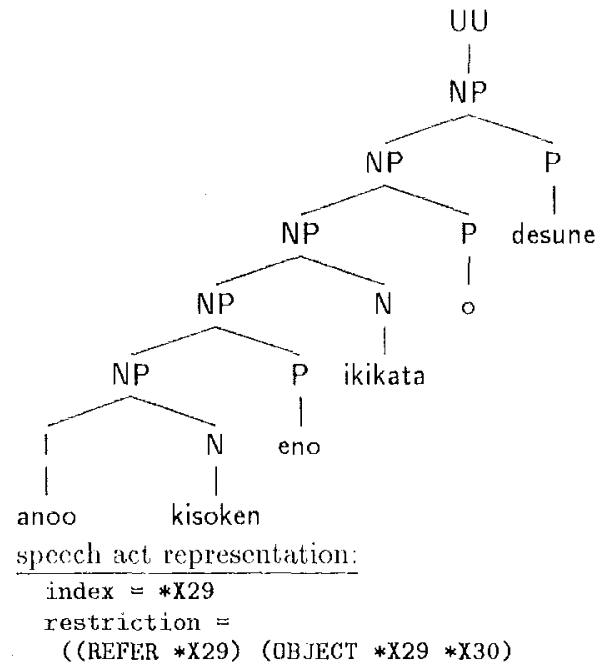

(AGFNT *X29*X31) (SPLAKER *X31)

(BASIC-RESEARCH-LABS $* \times 32$ )

(DFSTINATION $* X 30 * \mathrm{X} 32$ )

$(\mathrm{HOW}-\mathrm{TO}-\mathrm{GO} * \mathrm{X3O})$ )

- Utterance 4: 'oshic teitadaki tai ndesukedo' (I'd like you to tell me it)

parse tree:

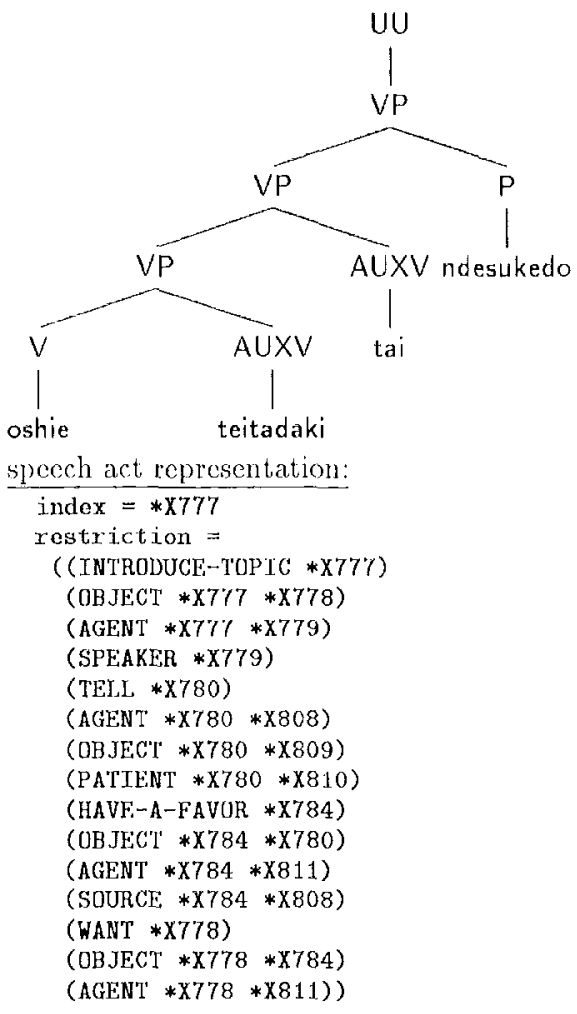

\section{CONCLUSION}

Wo have developed a grammar, called (irtuss-J, for handling distinctive phenomena in spontaneons: speech. 'The grammatical analysis of spontaneous speech is useful in combining the fruits of dialogue understanding research and those of speech processing research. As described earlier, Grass-J is used as the grammar for the experimental systems Enscmble/Trio-1 and Finsemble/Quartet-1, which are based on the Finsemble Model. It enables the processing of several kinds of spontancous specch, such as that lacking particles.

We focused on processing transcripts because a grammar and an analysis method for spontaneous speech can be combined with speech processing systems more accurately than can those for written langutages.

Finally, although we focused only on Japanese spontancous speech, most of the techniques described in this paper can also be used to analyze spontaneous speech in other languages.

\section{ACKNOWLEDGEMETNS}

We thank Chung Pai Ling, Yuiko Otsuka, Miyoko Sou, Kaeko Matsurawa, and Smae Nagata, for helping us analyzo dialogue datia. 


\section{REFERENCES}

Allen, J. F., \& Perrault, C. R. (1980). Analyzing Intention in Utterances. Artificial Intelligence, $15,143-178$.

Arita, H., Kogure, K., Nogaito, I., Maeda, H., \& lida, H. (1993). Media-Dependent Conversation Manners. In StG-NL-61, Information Processing Society of Jupan. (in Japanese).

Bcar, J., Dowding, J., \& Shriberg, L. (1992). Integrating Multiple Knowledgc Sources for the Detection and Correction of Repairs in HumanComputer Dialog. In $A C L-92$, pp. 56-63.

Clark, II. H., \& Wilkes-Gibbs, D. (1990). Referring as a Collaborative Process. In Cohen, P. R. Morgan, J., \& Pollack, M. F. (Fiss.), Intentions in Communication, pp. 463-493. MIT Press.

Cohen, P. R., Morgan, J., \& Pollack, M. E. (Eds.). (1990). Intentions in Communication. MI'I Press.

Den, Y. (1993). A Study on Spoken I)ialogue Grammar. SIG-SLUD-9902-5, Japanese Socicty of AI, 33 40. (in Japanese)

Gunji, 'I'. (1986). Japanese Phrase Structure Grammar. Reidcl, Dordrecht.

Ilayes, P. J., Hauptmann, A. G., Carbonell, J. G., \&. Tomita, M. (1986). Parsing Spoken I,anguage: A Semantic Caseframe Approach. In COLING86, pp. $587 \cdot 592$.

Hosaka, J., 'Takezawa, T., \& Ehara, 'T'. (1991). Constructing Syntactic Constraints for Speech Recognition using Empirical 1)ata. In $S / G-N L$ 83, Information Processing Socicty of Japan, pp. 97-104. (in Japanesc).

Katagiri, Y. (1993). Dialogue Coordination Functions of Japanese Sentence-Iinal Particles. In Proceedings of International Symposium on Spoken Dialogue, pp. 145 148.

Kawamori, M. (1991.). Japanese Sentence Final Particles and Fpistemic Modality. SIG-NI,-84, Infor mation Processing Society of Japan, 11-48. (in Japancse).

Kay, M. (1980). Nlgorithm Schemata and Data Structures in Syntactic Processing. Tech. rep. CSL80-12, Xerox PARC.

Kogure, K., Shimazu, A., \& Nakano, M. (1994), PlanBased Utterance Understanding. In Proceedings of the 48 th Conference of Information Processing Society of Japan, Vol. 3, pp. 189-190. (in Japanese).

Langcr, H. (1990). Syntactic Normalization of Spontaneous Spech. In COLING-90, pp. 180-183.

Levelt, W. J. M. (1989). Speaking. MIT Press

Mellish, C. (1989). Some Chart-Based l'cechniques for P'arsing Ill-Formed Input. In $A C L-89$, pp. 102 . 109.

Nagata, M., \& Kogure, K. (1990). HPSG-Based Lattice Parser for Spoken Japanese in a Spoken Language 'Translation System. In $E C A I-90, \mathrm{pl}$. $461-466$.

Nakano, M. (1991), Constraint Projection: An Ffflicient Treatment of Disjunctive Featurc Descriptions. In $A C L-91, \mathrm{pp} .307-314$
Nakatani, C., \& Wirschberg, J (1993). A Speech-First Model for Repair I)etection and Correction. In ACL-93, pp. 46. 53.

Okada, M. (1991). A Unification-Grammar-Directed One-Pass Search Algorithm for Parsing Spoken Language. In Proceedings of ICASSP-91.

Otsuka, II., \& Okada, M. (1992). Incremental lilaboration in Generating Spontaneous Speech. SIC NLC.92-41, Institute of blectronics, Information and Communication Lngineers. (in Japanese).

Sadanobu, T., \& Takubo, Y. (1993). The J)iscourse Management Function of Fillers-a case of "eeto" and "ano(o)"-. In Proceedings of Inlernational Symposium on Spoken Dialog, pp. 271-274.

Shicber, S. M. (1986). An Introduction to UnificationBased Approaches to Grammar. CSLI Jecture Notes Series No. 4. Stanford: CSLI.

Shimazu, A., Kawamori, M., \& Kogure, K. (1993a). Analysis of Interjectory Responses in Dialogue. SIG NLC-93-9, Institute of Electronics, Information and Comunication Engineers. (in Japanese).

Shimazu, A., Kogure, K., \& Nakano, M. (1993b). An Lxperimental Distributed Natural T,anguage Processing System and its Application to Robust Processing. In Proccedings of the Symposium on Implementation of Nalural language Processing. Institute of Flectronics, Information and Communication Engineers/Japan Society for Software Scionco and Technology. (in Japanesc).

'Lakeshita, A., \& Fukunaga, H. (1991). Morphological Analysis for Spoken Language. In Procecdings of the 42 nd Conference of information Processing Society of Japan, Vol. 3, pp. 5. 6. (in Japanese).

Thomason, R. Ir. (1990). Accommodation, Meaning, and Implicature: Interdisciplinary Foundations for Pragmatics. In Cohen, P. R., Morgan, J., \& Pollack, M. E. (Eds.), Intentions in Communication, pp. 325-364. MIT Press.

Young, S. R., Hauptmann, $\Lambda$, G., Ward, W. H. Smith, F. 'I., \& Werner, P'. (1989). High Level Knowledge Soures in Usable Speech Recognition Systems. Communication of the $A C M$, $32(2), 183 \cdot 194$ 